\title{
Regionalization Does Not Equal Integration
}

\author{
INTRODUCTION
}

\author{
Adalsteinn D. Brown, DPнгL \\ Editor-in-Chief, Healthcare Papers \\ Director, Institute of Health Policy, Management and Evaluation \\ University of Toronto \\ Toronto, ON \\ Peter W. T. Pisters, MD \\ President and CEO, University Heath Network \\ Toronto, ON \\ C. David Naylor, MD, DPнгц \\ Department of Medicine and Institute of Health Policy, Management and Evaluation \\ University of Toronto \\ Toronto, ON \\ $\propto$
}

The contributions to this issue of Healthcare Papers provide a clear picture of the strengths and weaknesses of regionalization efforts. The guest editor for this issue - Professor Greg Marchildon - summarizes the current environment well when he states that, for want of something better, "regionalization remains the most viable means" to the improvement of healthcare system performance in Canada.

It seems that governments agree with this summary. The last decade has seen a spate of efforts to rework the architecture of Canada's healthcare systems. Provinces have regrouped the sets of providers included under regions (e.g., Quebec), redrawn regional boundaries (e.g., Alberta) or restated the accountabilities of regions (most provinces). Even Ontario for a long time the control group for Canadian experiments in regionalization - created the local health integration networks (LHINs). Ten years later, Ontario is considering even more sweeping consolidation of healthcare providers and powers under the LHINs.

To justify regionalization, policy makers commonly cite a greater focus on public health and health promotion, cost-saving consolidation of administrative processes and the promise that health services will finally reflect how patients receive care from different providers as 
they move through the system. Unfortunately, policy makers sometimes conflate regionalization - the lumping of providers under regional governance - with integration or the organization of care around patient needs. Like regionalization, integration may sound like jargon. However, many, if not most, patients in Canada still experience the healthcare system as a series of disconnected siloes. Providers too are frustrated when lack of integration undercuts the ideal of coordinated and continuing care for the changing health needs of individual patients. When care providers work together in a way that aligns their professional practices, information systems, incentives and cultures, they can provide health services faster, more reliably and with better outcomes. For the patient, that means fewer tests and unnecessary visits, better communication and a lower risk for medical errors.

Regionalization can support integration, but it is neither sufficient nor necessary to improve integration and health system performance. In this issue, Yves Bergevin and colleagues provide a comprehensive review of global experiences in regionalization and conclude with seven lessons that talk about how regionalization turns into integration and a clearer focus on the patient. These lessons emphasize that the best regionalized systems do more than bring budgets together. They also change the way monies flow, creating incentives for integrated care, opportunities for reinvesting based on measured performance and a necessity for stronger clinical leadership. They also point, somewhat painfully, to the fact that we need a second phase of regionalization reforms that go well beyond current debates about who controls whom and who gets to hold the money.

Here is what that second phase of reform might ideally involve:

First, real integration needs to occur. That's especially true when so many patients receive sophisticated and comprehensive healthcare services from multiple providers. Better-coordinated care will result in improved quality, enhanced patient experience and lower societal costs. A rapid way forward is for provinces to encourage and financially support new consortia of institutions and other local care providers that grow from coalitions of providers focused on meeting community needs. These networks of providers will thrive if they are big enough to provide comprehensive care and achieve economies of scale, and if the right incentives are put in place.

That brings us to reinvestment for performance. Each year that a network improves care for patients in a meaningful way, it should be able to reinvest these savings back into further system improvements. However, to ensure that the improvements are real and corners aren't being cut, ministries, regions and providers must collaborate to generate detailed public scorecards for each network - scorecards that focus on outcomes like health status, patient experience and safe care. Making quality improvement the first job of regions is a key lesson from the US experience with accountable care organizations (ACOs) that is too often neglected when we try to simultaneously juggle cost control and quality in Canada.

These changes would go a long way to rewarding achievement and creating a more innovative and dynamic culture within our provincial healthcare systems. They play to the best side of Canada's doctors, nurses and other healthcare professionals by recognizing and rewarding better care rather than just volumes and costs of services. And, if these changes focus more attention upstream on population health status, then health promotion and maintenance in turn may get greater attention.

The third step is also crucial. Healthcare systems across the country urgently need to strengthen what is often called 
"clinical governance." In top-performing health systems, doctors, nurses and other health professionals are constantly and closely engaged in leading efforts to improve care. They receive training, have access to data on care and are supported in improvement efforts, so that quality improvement is seen as part of the clinicians' jobs, not just an extra task to be pursued when the last patient has been seen. These systems also invest in leadership development, so that more doctors, nurses and other providers can take a big picture view, use data wisely, embrace innovations in healthcare and generate and test their own ideas for improving the system.
Moving from regionalization to integration is tough sledding at a time when most ministries of health face dramatic and continuing financial pressures. More than a few provinces are at loggerheads with their doctors as they try to change the organization of care and limit physician expenditure. Creating new structures may be part of the necessary reforms to our system, but it should not be the only part. The faster we can get to that next phase of reform with its possibilities of better integration, smart reinvestment and reporting, and stronger clinical governance, the faster Canada can regain its leadership in healthcare.

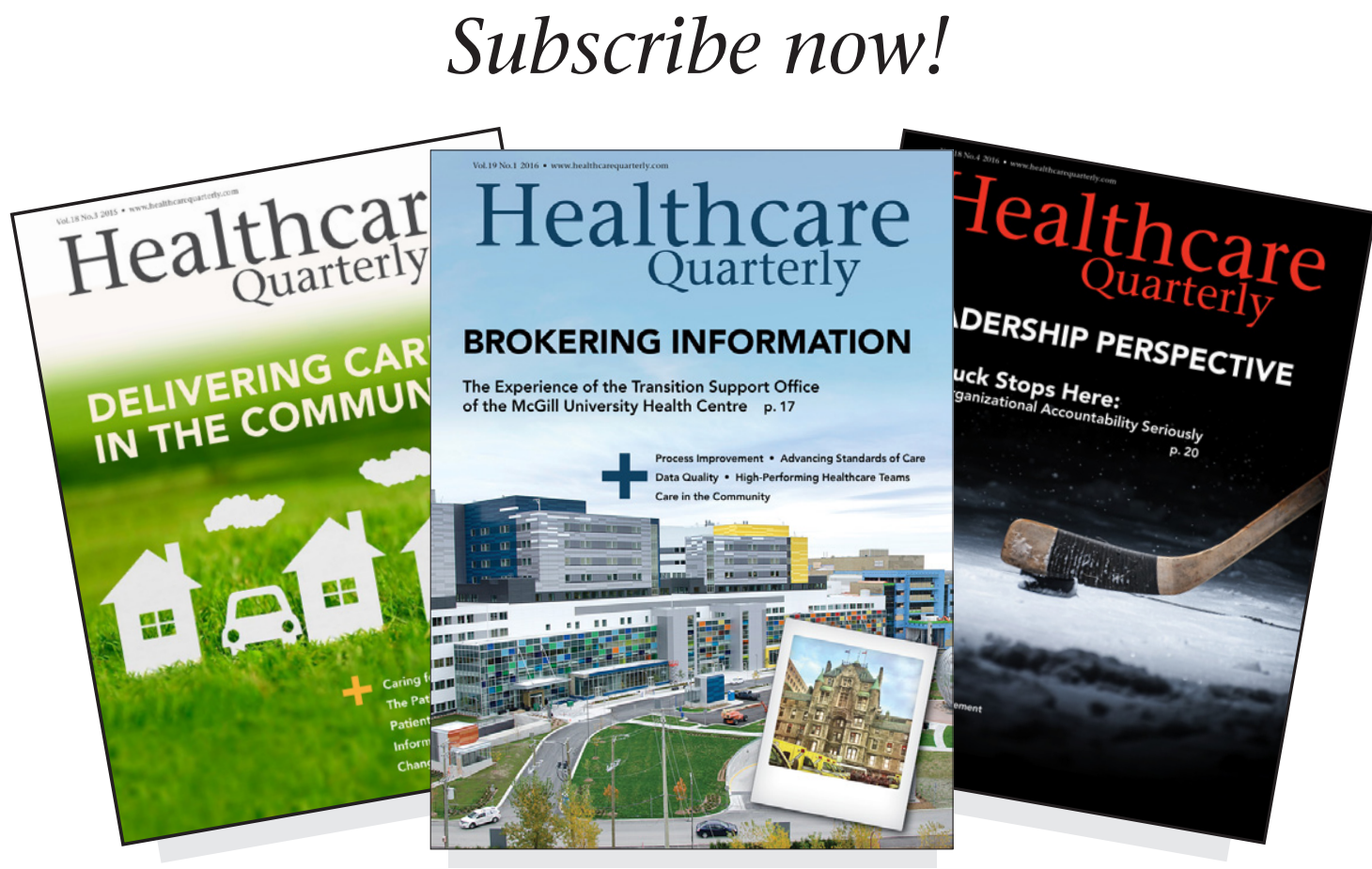

Healthcare Quarterly recognizes, nurtures and champions excellence in the Canadian healthcare system. Its objective is to document and disseminate leading practices in health service delivery and policy development. Excellence is achieved through constant innovation, motivated people and inspired leadership at all levels of the organization. Healthcare Quarterly helps Canadian health system managers anticipate and respond to changing environments, demands and mandates. 